\title{
Crossover from phonon- to photon-mediated charge transport observed in metal-cluster compounds
}

\author{
J. A. Reedijk, L. J. Adriaanse, H. B. Brom, and L. J. de Jongh \\ Kamerlingh Onnes Laboratory, Leiden University, P.O. Box 9506, 2300 RA Leiden, The Netherlands \\ G. Schmid \\ Institut für Anorganische Chemie, Universität GH Essen, Universitätsstrasse 5-7, D-45117 Essen, Germany
}

(Received 12 January 1998)

\begin{abstract}
We have studied the frequency-dependent conductivity of randomly packed aggregates of ligand-stabilized, monodisperse metal clusters from dc to the infrared over 12 decades in frequency. At low frequencies the ac conductivity of these compounds is dominated by phonon-assisted hopping between localized states, and shows scaling behavior as a function of temperature and frequency. Due to the unique structure of these cluster aggregates, the transition from phonon- to photon-mediated charge transport is clearly observed. The data are found to be in excellent quantitative agreement with the two-site tunneling model. [S0163-1829(98)50324-4]
\end{abstract}

Charge transport in disordered systems, where conduction proceeds by hopping between localized states, has been a widely studied subject over the last decades. A powerful method to study hopping transport is to perform conductivity experiments where both temperature and applied frequency are varied. In this way, not only the various theoretical hopping models can be tested, ${ }^{1}$ but also insight in the structural properties of the material at mesoscopic length scales may be gained. ${ }^{2,3}$

Hopping conduction between localized states $i$ and $j$ can take place when the overlap between the wave functions $\phi$ at sites $i$ and $j$, i.e., $I_{i j}=\int d \mathbf{r} \phi^{*}\left(\mathbf{r}-\mathbf{r}_{i}\right) \mathcal{H} \phi\left(\mathbf{r}-\mathbf{r}_{j}\right)$ with $\mathcal{H}$ the Hamiltonian of the system, is nonzero. This overlap, together with the site energy difference $E_{j}-E_{i}$, determines the energy needed to make a hop. When the field strength $\mathbf{E}$ and the applied frequency $\omega$ are small, this hop energy has to be supplied by lattice vibrations, and the transport proceeds by phonon-assisted hopping. At high frequencies, however, the photon energy $\hbar \omega$ may become sufficient to induce a resonant tunneling transition. This phononless tunneling process will start to contribute to the conductivity when $\hbar \omega$ becomes comparable to the overlap energy $I_{i j} \cdot{ }^{4}$

A class of materials that is often used for studying charge transport in disordered systems are random composites of metal particles in an insulating host. When the conducting filler fraction $p$ is lower than the percolation threshold $p_{\mathrm{c}}$, the electron wave functions are localized on the metal particles, and transport occurs via tunneling through the insulating barriers. Discontinuous films and granular metals with relatively high tunneling probabilities due to a sufficient dense packing of the metal particles, have been frequently investigated in dc conductivity experiments, ${ }^{5-7}$ while the low-frequency ac conductivity of these compounds has been relatively little studied. ${ }^{8,9}$ Diluted insulator/metal composites $\left(p \ll p_{c}\right)$ have often been used in far-infrared (i.e., around 1 $\mathrm{THz}$ ) absorption spectroscopy, ${ }^{10-12}$ with the idea that experiments on diluted systems should be more sensitive to the properties of the individual metal particles.

In this work, we report on the frequency-dependent conductivity of several samples consisting of small metal nano- particles stabilized by ligand shells. These unique materials enable us to present a dielectric study of small metal particle systems which runs almost continuously from the lowfrequency region into the infrared. The data clearly show a gradual transition from the phonon-assisted hopping characteristics at low frequencies to the photon-induced resonant tunneling processes predicted at far-infrared frequencies.

\section{EXPERIMENTAL}

All samples studied are well-defined stoichiometric metal cluster compounds, in which monodisperse metal cluster cores are surrounded by stabilizing ligand shells. ${ }^{13,14}$ The ligands are organic molecules like phenantroline or chinchonidine. ${ }^{13}$ The metal cores consist of a central atom surrounded by a number of shells of fcc packed atoms. The core diameters are $1.4 \mathrm{~nm}$ for the two shell Au cluster (abbreviated as Au-2), $2.0 \mathrm{~nm}$ for the four shell Pt (Pt-4), and $3.5 \mathrm{~nm}$ for a $50 / 50$ mixture of seven and eight shell Pd (Pd$7 / 8$ ) clusters. The metal volume fractions are $p \approx 20 \mathrm{vol} \%$ for Pt-4 and two Au-2 samples, and $p \approx 40$ vol $\%$ for Pd$7 / 8$. Diffraction experiments indicate that the densely packed clusters do not form a regular lattice, but are randomly stacked. $^{13}$

The frequency-dependent complex conductivity was studied in the frequency range $5 \mathrm{~Hz}-3 \mathrm{THz}$. To cover this huge range in frequency, several experimental techniques were combined. ${ }^{3}$ For the capacitive method employed below 1 $\mathrm{GHz}$, the sample was pressed between two electrodes. Between $200 \mathrm{MHz}$ and $18 \mathrm{GHz}$, we used an open ended coaxial probe. The frequency range $30-100 \mathrm{GHz}$ was covered by means of a filled waveguide technique, and between 0.2 and $0.6 \mathrm{THz}$ by a quasioptical setup allowing the measurement of both attenuation and phase of the transmitted signal without the application of contacts ${ }^{15}$. Between 0.3 and $3 \mathrm{THz}$, we used a Fourier transform infrared spectrometer.

Measurements at frequencies from dc up to $13 \mathrm{MHz}$ were done between room temperature and $4 \mathrm{~K}$. Four-point dc measurements indicated that, using pressed brass contacts, con- 


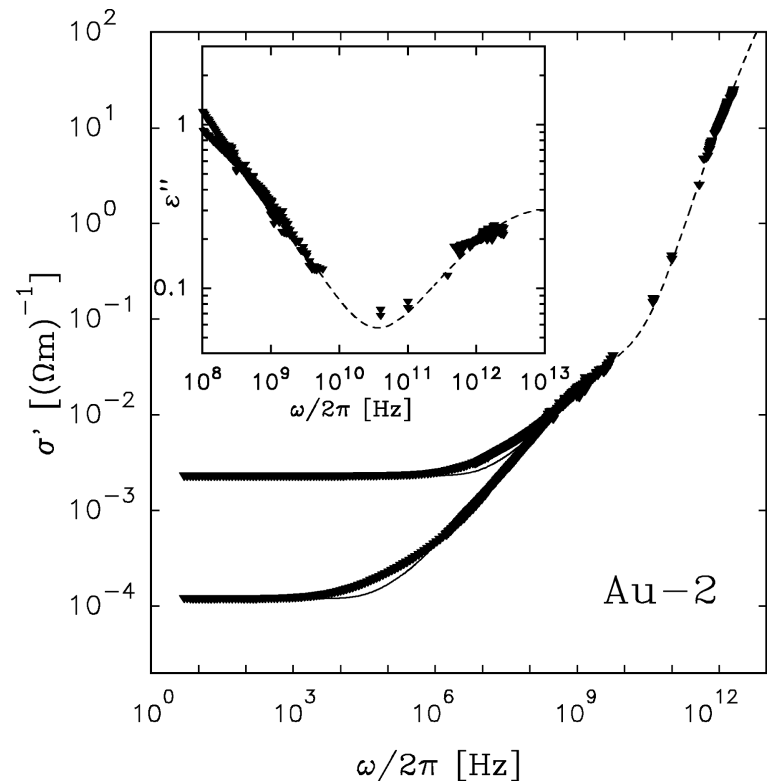

FIG. 1. $\sigma^{\prime}(\omega)$ at $300 \mathrm{~K}$ between $5 \mathrm{~Hz}$ and $3 \mathrm{THz}$ for two Au-2 samples with different ligands; the sample with the thicker ligands has lower $\sigma^{\prime}$ at low $\omega$. The drawn lines represent fits with the effective medium model $(\omega / 2 \pi<100 \mathrm{MHz})$; a fit to the two-site model, including phonon- and photon-assisted tunneling, is indicated by the dashed line $(\omega / 2 \pi>100 \mathrm{MHz})$. In the inset, the loss function $\epsilon^{\prime \prime}(\omega)=\sigma^{\prime}(\omega) / \epsilon_{0} \omega$ is shown for the two Au-2 samples, together with the fit to the two-site model (dashed line).

tact resistances were small ( $\leqslant 5 \%$ of the sample resistance). Above $13 \mathrm{MHz}$, all experiments were performed at ambient temperature.

\section{RESULTS AND DISCUSSION}

In an earlier study, the $T$ dependence of the dc conductivity of these compounds was found to be well described by variable-range hopping. ${ }^{16}$ Figure 1 shows the frequencydependent conductivity (the inset gives the related imaginary part of the dielectric constant) at room temperature of two Au-2 samples with different ligands over the entire frequency range $5 \mathrm{~Hz}-3 \mathrm{THz}$. The sample with slightly thicker ligands has lower conductivity at low frequencies. Dashed and drawn lines are fits based upon, respectively, two site and multiple hopping models (see below).

In Fig. 2, the frequency-dependent conductivity of the cluster compound Pd-7/8 is plotted, again fitted with a multiple hopping model discussed below. Data on this sample (which is well suited to study low-frequency scaling, since its high dc conductivity allows experiments and scaling down to helium temperatures) were taken up to $13 \mathrm{MHz}$ at temperatures between $4 \mathrm{~K}$ and $300 \mathrm{~K} .{ }^{17}$ In this temperature range, $\sigma_{\mathrm{dc}}$ varies over seven orders of magnitude. Using scaled conductivity $\sigma^{\prime}(\omega, T) / \sigma_{\mathrm{dc}}(T)$ and scaled frequency $\omega / \sigma_{\mathrm{dc}}(T)$, data sets of all temperatures fall on top of each other. The same scaling behavior is seen in the $T$-dependent low-frequency data on all other compounds studied (including $\mathrm{Au}-2 \mathrm{)}$ and the scaling curves are very similar for all cluster compounds. ${ }^{16}$

Many hopping models predict a scaling of $\sigma^{\prime}(\omega, T) .{ }^{18-21}$ Often the frequency is scaled by a characteristic frequency

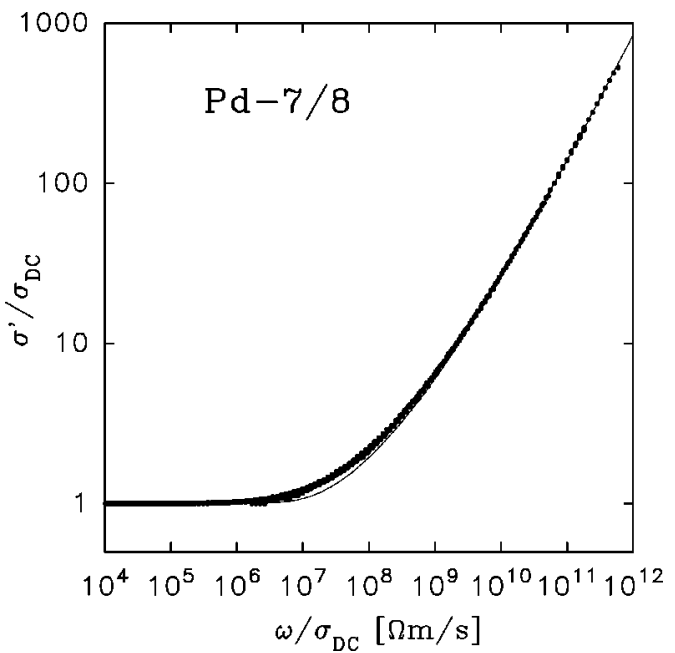

FIG. 2. Scaled conductivity $\sigma^{\prime}(\omega) / \sigma_{\mathrm{dc}}$ vs scaled frequency $\omega / \sigma_{\mathrm{dc}}$ of the Pd-7/8 sample. Data taken at $5 \mathrm{~Hz}<\omega / 2 \pi<13 \mathrm{MHz}$ and temperatures $4 \mathrm{~K}<T<300 \mathrm{~K}$ collapse onto a single curve. The solid line represents a fit to the effective medium prediction.

$\omega^{*}$, which is proportional to $T \sigma_{\mathrm{dc}}(T) .{ }^{18}$ However, the $T$-independent scaling $\omega^{*} \propto \sigma_{\mathrm{dc}}(T)$ observed here is frequently found experimentally and, e.g., expected in a diffusion model when the carrier density $n \propto T,{ }^{3}$ which is the case for hopping transport near the Fermi level. ${ }^{22}$

Bryksin $^{23}$ has calculated the ac conductivity for spatially random systems with nearest-neighbor tunneling in an effective medium approximation. In this model, a wide distribution of hopping times due to complete spatial randomness is assumed. This theory has later been extended ${ }^{21}$ to systems where conduction proceeds by means of variable-range hopping. Both models predict the conductivity in the low and intermediate frequency regime, where multiple hopping is dominant, to obey the relation (where $\tilde{\sigma}=\sigma / \sigma_{\mathrm{dc}}$ is the normalized complex conductivity and $\tilde{\omega}$ is the scaled frequency $\left.\omega / \omega^{*}\right)$

$$
\tilde{\sigma} \ln \tilde{\sigma}=i \tilde{\omega} .
$$

Solving this equation numerically, $\sigma^{\prime}(\widetilde{\omega})$ can be computed. Together with the room-temperature data on the $\mathrm{Au}-2$ samples, the theoretical curve for $\sigma^{\prime}(\omega)$ is plotted in Fig. 1 (solid line). In Fig. 2, the same theoretical curve is plotted together with the scaled $\sigma^{\prime}(\omega)$ data for Pd-7/8. In both compounds, a clear deviation between data and prediction is present at frequencies around the "onset" frequency $\omega_{0}$, where $\sigma^{\prime}(\omega)$ starts to deviate from the dc value. At higher frequencies data and theory agree well. In all cluster compounds studied, the discrepancy around $\omega_{0}$ is found. ${ }^{24}$ Similar deviations have also been observed in other experiments ${ }^{25,26}$ and computer simulations. ${ }^{27}$

The failure of effective medium models ${ }^{28-30}$ at frequencies $\omega \approx \omega_{0}$ can be understood by realizing that lowfrequency transport is dominated by multiple hopping over a large number of connected sites, known as the "infinite cluster" in percolation theory. ${ }^{31}$ The complex, inhomogeneous structure of this percolating network is not incorporated in the effective medium theory of Ref. 21. Using arguments 
from percolation theory, Hunt ${ }^{32}$ showed that relaxation rates of assemblies of connected sites can be orders of magnitude slower than individual pair relaxation rates, and hence effectively increase the conductivity for low frequencies $\omega \leqslant \omega_{0}$.

At higher frequencies $\omega>\omega_{0}$, where transport occurs only on small groups of connected sites and hence the slow relaxation rates are irrelevant, the close agreement between the data and the effective medium model (see Fig. 2) indicates that in the material no structure is present at mesoscopic length scales, since this would result in a specific frequency dependence of the conductivity at $\omega>\omega_{0},{ }^{2,3}$

\section{$\sigma^{\prime}(\omega)$ AT HIGH FREQUENCIES}

In order to display more explicitly the features of the high frequency conductivity data, the loss function $\epsilon^{\prime \prime}(\omega)$ $=\sigma^{\prime}(\omega) / \epsilon_{0} \omega$ is plotted in the inset of Fig. 1 for the two $\mathrm{Au}-2$ samples in the high frequency range $(100 \mathrm{MHz}-3$ $\mathrm{THz}$ ). In this frequency range, the two data sets fall on top of each other, indicating that ligand effects are negligible at high frequencies. With increasing $\omega, \epsilon^{\prime \prime}$ first decreases, and then starts to increase around $\omega / 2 \pi=50 \mathrm{GHz}$. The same minimum in the high-frequency loss function is found in the four-shell Pt compound (not shown). Below we shall argue that this minimum in $\epsilon^{\prime \prime}$ indicates the crossover from phonon- to photon-induced tunneling transport.

In a system of isolated small metal particles, classical electromagnetic theories predict $\sigma^{\prime}(\omega) \propto \omega^{2}$ at far-infrared frequencies, due to the low-frequency tail of a surface plasmon resonance of the conduction electrons. ${ }^{10}$ An approximate $\sigma^{\prime} \propto \omega^{2}$ behavior at for far-infrared (FIR), frequencies has indeed been found many times in samples of metal particles diluted in a nonabsorbing host. ${ }^{10-12}$ However, the absolute value of the absorption was always orders of magnitude larger than the predictions for intraparticle relaxation.

Similar features are seen in the high frequency data $\left(\omega / 2 \pi>10^{11} \mathrm{~Hz}\right)$ of the Au-2 and Pt-4 compounds. Although the data can be approximaly fitted with $\sigma^{\prime} \propto \omega^{2}$, i.e. $\epsilon^{\prime \prime} \propto \omega$, the absolute value of the measured conductivity ( $\sigma^{\prime}$ $\approx 10 \mathrm{~S} / \mathrm{m}$ at $1 \mathrm{THz}$ ) is almost four orders of magnitude larger than expected from intracluster dipole absorption. ${ }^{33}$

To explain the earlier observed strong FIR absorption in metal particle systems, several models were proposed in a paper by Curtin and Ashcroft. ${ }^{34}$ One of the possibilities suggested was an enhancement of the conductivity due to photon-induced tunneling between metal particles. In this model, the FIR conductivity for an assembly of metal particles, weakly coupled by tunnel junctions, is given by $\sigma^{\prime}$ $\propto \omega^{2}$ at low frequencies, with a prefactor comparable to the experimentally found values. In this model, relatively high interparticle tunneling probabilities are assumed. Such high tunneling probabilities can only occur in diluted systems when, due to strong inhomogeneities, regions of densely packed metal particles are present. Indeed, clustering of particles into dense regions has been observed many times in experimental diluted small metal particle systems. ${ }^{11}$

In a more general treatment of hopping conduction between localized states in disordered systems, Böttger and Bryksin calculated the conductivity in a two-site approximation, ${ }^{19}$ valid for high frequencies where the multiple hopping process is negligible. Two processes were found to contribute to the conductivity: "classical" phononassisted tunneling, dominant at lower frequencies, and resonant phononless tunneling, dominating at high $\omega$. The phonon-assisted contribution was found to follow:

$$
\sigma_{\text {phonon }}^{\prime}(\omega)=\frac{\pi^{4}}{384} e^{2} \omega k_{\mathrm{B}} T \alpha^{-5} n_{0}{ }^{2} \ln ^{4}\left(\nu_{\mathrm{ph}} / \omega\right),
$$

where $n_{0}$ is the density of states at the Fermi level, $\alpha$ is the inverse electron localization length, and $\nu_{\mathrm{ph}}$ is the phonon "attempt" frequency.

The probability of a photon-induced tunneling transition between states $i$ and $j$ depends strongly on the overlap energy $I_{i j}=\int d \mathbf{r} \phi^{*}\left(\mathbf{r}-\mathbf{r}_{i}\right) \mathcal{H} \phi\left(\mathbf{r}-\mathbf{r}_{j}\right)$, which can be conveniently written as $I_{i j}=I_{0} e^{-\alpha\left|\mathbf{r}_{i}-\mathbf{r}_{j}\right|}$. Integrating over all pairs, Böttger and Bryksin ${ }^{19}$ derive for the phononless contribution to the conductivity

$$
\sigma_{\text {photon }}^{\prime}(\omega)=\frac{\pi^{2}}{6} e^{2} \hbar \omega^{2} \alpha^{-5} n_{0}{ }^{2} \ln ^{4}\left(2 I_{0} / \hbar \omega\right) .
$$

Apart from a small numerical factor, this coincides with an earlier result of Mott, derived for zero temperature. ${ }^{4}$ The conductivity at high frequencies is now given by the sum of the two contributions, i.e., $\sigma^{\prime}(\omega)=\sigma_{\text {phonon }}^{\prime}+\sigma_{\text {photon }}^{\prime}$. The data on the cluster compounds can be adequately fitted with this equation, as is shown in Fig. 1 for the Au-2 samples. For this fit, three parameters $\left(\nu_{\mathrm{ph}}, I_{0}\right.$, and $\left.\alpha^{-5} n_{0}{ }^{2}\right)$ were varied. From the fit for $\mathrm{Au}-2$, we find for the attempt frequency $\nu_{\mathrm{ph}}=8 \times 10^{12} \mathrm{~Hz}$, which lies in the range of usually suggested values of $10^{12}$ to $10^{14} \mathrm{~Hz}$. For the overlap prefactor we find $I_{0}=1.2 \mathrm{eV}$. The corresponding rate $I_{0} / \hbar$ should be interpreted as the attempt frequency for photon-assisted hopping, i.e., as the electronic collision rate within the clusters. This collision rate can be estimated by $v_{\mathrm{F}} / d$ with $d=1.4 \mathrm{~nm}$ for Au-2, giving $v_{\mathrm{F}}=2 \times 10^{6} \mathrm{~m} / \mathrm{s}$, close to the Fermi velocity of bulk gold.

Comparing the absolute value of the measured conductivity with the predictions for the two-site model, we find that they coincide when $n_{0}^{2} \alpha^{-5}=10^{4} \mathrm{eV}^{-2} \mathrm{~cm}^{-1}$. With reasonable esimates $n_{0}=6 \times 10^{20} \mathrm{eV}^{-1} \mathrm{~cm}^{-3}$ and $\alpha^{-1}=3 \AA{ }^{35}$ full quantitative agreement with the two-site model is found for frequencies $100 \mathrm{MHz}<\omega / 2 \pi<3 \mathrm{THz}$.

We note that in the two-site approximation, the distribution of hopping lengths $p(R)$ follows from a completely random site distribution to be $p(R) \propto R^{2}$. In the cluster compounds, the hopping length distribution will be different especially at small $R$, since the ligand shells prevent the clusters to be separated by less than $2 d$, where $d$ is the ligand shell thickness, i.e., $p(R)=0$ for $R<2 d$. We numerically found that the changed hopping length distribution has negligible influence on the conductivity as long as the "characteristic pair separation" $R(\omega)$ [given by $(2 \alpha)^{-1} \ln \left(\nu_{\mathrm{ph}} / \omega\right)$ for the phonon contribution, and $\alpha^{-1} \ln \left(2 I_{0} / \hbar \omega\right)$ for the photon contribution ${ }^{19}$ ] exceeds the minimum pair separation $R$ $=2 d$. For the Au- 2 samples, $d \approx 3.5 \AA$, i.e., Eq. (2) may be used for $\omega / 2 \pi<12 \mathrm{GHz}$, and Eq. (3) is valid for $\omega / 2 \pi$ $<50 \mathrm{THz}$.

In summary, the frequency-dependent conductivity data of the metal cluster compounds studied clearly show a cross- 
over from phonon-assisted hopping at low frequencies to photon-induced transport around $\mathrm{THz}$ frequencies. The data taken at low frequencies, where multiple hopping dominates, can be fitted adequately with an effective medium model. The superlinear rise of $\sigma^{\prime}(\omega)$ at high frequencies is explained with a photon-assisted tunneling model. The parameters found from the phonon- and photon-mediated conductivities in the two site regime, which at room temperature starts above $100 \mathrm{MHz}$, are fully consistent.

\section{ACKNOWLEDGMENTS}

This work was part of the research program of the "Stichting voor Fundamenteel Onderzoek der Materie", (FOM) which is partially supported by the "Nederlandse Organisatie voor Wetenschappelijk Onderzoek' (NWO). The discussions with H.C.F. Martens, and the assistance of G.A. van Albada with the FIR experiments are gratefully acknowledged, as is the support of the European Community under the HCM program.
${ }^{1}$ See, e.g., M.P.J. van Staveren, H.B. Brom, and L.J. de Jongh, Phys. Rep. 208, 1 (1991).

${ }^{2}$ M. Ben-Chorin, F. Möller, F. Koch, W. Schirmacher, and M. Eberhard, Phys. Rev. B 51, 2199 (1995).

${ }^{3}$ L.J. Adriaanse, J.A. Reedijk, P.A.A. Teunissen, H.B. Brom, M.A.J. Michels, and J.C.M. Brokken-Zijp, Phys. Rev. Lett. 78, 1755 (1997).

${ }^{4}$ N.F. Mott and E.A. Davies, Electronic Processes in NonCrystalline Materials (Oxford University Press, Oxford, 1971), Sect. 2.4.

${ }^{5}$ B. Abeles, Ping Sheng, M.D. Coutts, and Y. Arie, Adv. Phys. 24, 407 (1975).

${ }^{6}$ T. Chui, G. Deutscher, P. Lindenfeld, and W.L. McLean, Phys. Rev. B 23, 6172 (1981).

${ }^{7}$ O. Entin-Wohlman and Z. Ovadyahu, Phys. Rev. Lett. 56, 643 (1986).

${ }^{8}$ R.B. Laibowitz and Y. Gefen, Phys. Rev. Lett. 53, 380 (1984).

${ }^{9}$ Y. Song, T.W. Noh, S.-I. Lee, and J.R. Gaines, Phys. Rev. B 33, 904 (1986).

${ }^{10}$ G.L. Carr, R.L. Henry, N.E. Russell, J.C. Garland, and D.B. Tanner, Phys. Rev. B 24, 777 (1981).

${ }^{11}$ T.W. Noh, S.-I. Lee, and J.R. Gaines, Phys. Rev. B 33, 1401 (1986)

${ }^{12}$ Y.H. Kim and D.B. Tanner, Phys. Rev. B 39, 3585 (1989).

${ }^{13}$ Clusters and Colloids, edited by G. Schmid (VCH, Weinheim, 1994); G. Schmid and N. Beyer (unpublished).

${ }^{14}$ Physics and Chemistry of Metal Cluster Compounds, edited by L.J. de Jongh (Kluwer, Dordrecht, 1994).

${ }^{15}$ AB Millimetre MVNA S/N 21 User's Manual, Paris, France.

${ }^{16}$ L.J. Adriaanse, J.A. Reedijk, H.B. Brom, L.J. de Jongh, and G. Schmid, Z. Phys. D 40, 123 (1997).

${ }^{17}$ Due to the lack of sufficient sample, experiments on this compound could not be extended into the $\mathrm{THz}$ region.

${ }^{18}$ S. Summerfield, Philos. Mag. B 52, 9 (1985).

${ }^{19}$ H. Böttger and V.V. Bryksin, Hopping Conduction in Solids
(VCH Akademie-Verlag, Berlin, 1985).

${ }^{20}$ W. Schirmacher, Ber. Bunsenges. Phys. Chem. 95, 368 (1991).

${ }^{21}$ O. Bleibaum, H. Böttger, and V.V. Bryksin, Phys. Rev. B 54, 5444 (1996).

${ }^{22}$ V. Ambegaokar, B.I. Halperin, and J.S. Langer, Phys. Rev. B 4, 2612 (1971).

${ }^{23}$ V.V. Bryksin, Fiz. Tverd. Tela (Leningrad) 22, 2441 (1980) [Sov. Phys. Solid State 22, 1421 (1980)].

${ }^{24}$ When fitting the data with other effective-medium approximation predictions for systems with less wide jump frequency distributions, the discrepancy around $\omega_{0}$ remains.

${ }^{25}$ K.K. Bardhan and R.K. Chakrabarty, Phys. Rev. Lett. 72, 1068 (1994).

${ }^{26}$ A.R. Long, J. McMillan, N. Balkan, and S. Summerfield, Philos. Mag. B 58, 153 (1988).

${ }^{27}$ J.C. Dyre, Phys. Rev. B 49, 11709 (1994).

${ }^{28}$ Also other theoretical considerations (Refs. 29 and 30) indicate that the low-frequency hopping conductivity does not follow Eq. (1).

${ }^{29}$ V.V. Bryksin, Fiz. Tverd. Tela 26, 1362 (1984) [Sov. Phys. Solid State 26, 827 (1984)].

${ }^{30}$ J.C. Dyre and T.B. Schrøder, Phys. Rev. B 54, 14884 (1996).

${ }^{31}$ D. Stauffer and A. Aharony, Introduction to Percolation Theory (Taylor \& Francis, London, 1992).

${ }^{32}$ A. Hunt, Philos. Mag. B 64, 579 (1991); J. Non-Cryst. Solids 183, 109 (1995).

${ }^{33}$ Using Eq. (8) of Ref. 10 with $a=1.4 \mathrm{~nm}, f=0.2$, and $\sigma_{0}$ $=10^{7} \mathrm{~S} / \mathrm{m}$, we find $\sigma^{\prime} \approx 2 \times 10^{-3} \mathrm{~S} / \mathrm{m}$ at $1 \mathrm{THz}$.

${ }^{34}$ W.A. Curtin and N.W. Ashcroft, Phys. Rev. B 31, 3287 (1985).

${ }^{35}$ The density of states $n_{0}$ in Au-2 is estimated assuming a uniform band of width $W \approx 0.3 \mathrm{eV}$ (which is approximately equal to the charging energy of a cluster), in which all clusters contribute one state; this gives $n_{0}=6 \times 10^{20} \mathrm{~cm}^{-3} \mathrm{eV}^{-1}$. The localization radius $\alpha^{-1}$ is related to the binding energy to the cores via $E_{b}$ $=\hbar^{2} \alpha^{2} / 2 m$; when $\alpha^{-1}=3 \AA$, this binding energy $E_{b}=1 \mathrm{eV}$. 